\title{
Similarity Transformations of MAPs
}

\author{
ALLAN T. ANDERSEN, V.A. BARKER and BO FRIIS NIELSEN* \\ Department of Mathematical Modelling, Building 321, Technical University of \\ Denmark, DK-2800 Lyngby, Denmark
}

(Received 12 October 1998)

\begin{abstract}
We introduce the notion of similar Markovian Arrival Processes (MAPs) and show that the event stationary point processes related to two similar MAPs are stochastically equivalent. This holds true for the time stationary point processes too. We show that several well known stochastical equivalences as e.g. that between the $H_{2}$ renewal process and the Interrupted Poisson Process (IPP) can be expressed by the similarity transformations of MAPs. In the appendix the valid region of similarity transformations for two-state MAPs is characterized.
\end{abstract}

Keywords: Markovian Arrival Process; Similarity; Stochastical equivalence

\section{INTRODUCTION}

In this paper we consider similarity transformations of the matrices $\left(\boldsymbol{D}_{0}, \boldsymbol{D}_{1}\right)$ which describe the Markovian Arrival Process (MAP). The MAP has the generator $D=\left(D_{0}+D_{1}\right)$ which has stationary probability vector $\vec{\pi}$. The state transitions in $D_{1}$ are associated with arrivals while those in $\boldsymbol{D}_{0}$ are not. The interval stationary probability vector is given by $\vec{\phi}=\left(\vec{\pi} \boldsymbol{D}_{1} \vec{e}\right)^{-1} \vec{\pi} \boldsymbol{D}_{1}$. For general literature on MAPs see e.g. $[4,5]$. The notion of Stochastical Equivalence (SE) is an important one when considering point processes generated by different MAPs. Two different MAPs will in general not be identical in any reasonable probabilistic sense with respect to an arbitrary initial state. However, for certain combinations of initial conditions the point processes of

\footnotetext{
*Corresponding author. E-mail: bfn@imm.dtu.dk.
} 
several MAPs might very well have identical probabilistic behavior. It is most relevant to consider the time and interval stationary behavior. In the MAP setting the equivalence of the Interrupted Poisson Process (IPP) and the renewal process with an interarrival time distribution according to a two-phased hyper-exponential, $\mathrm{H}_{2}$, is the classical example of this construction.

\section{STOCHASTICAL EQUIVALENCE}

We give an important definition following the lines of [3].

DEFINITION 2.1 Two point processes are stochastically equivalent if for any $n \geq 1$ the joint distributions of the first $n$ intervals agree.

The joint Laplace-Stieltjes transformation $\Psi^{\mathrm{i}}\left(s_{1}, \ldots, s_{n}\right)$ of the first $n$ intervals in the interval stationary version of the MAP $\left(\boldsymbol{D}_{0}, \boldsymbol{D}_{1}\right)$ is given as

$$
\Psi^{\mathrm{i}}\left(s_{1}, \ldots, s_{n}\right)=\left(\vec{\pi} \boldsymbol{D}_{1} \vec{e}\right)^{-1} \vec{\pi} \boldsymbol{D}_{1}\left(s_{1} \boldsymbol{I}-\boldsymbol{D}_{0}\right)^{-1} \boldsymbol{D}_{1} \cdots\left(s_{n} \boldsymbol{I}-\boldsymbol{D}_{0}\right)^{-1} \boldsymbol{D}_{1} \vec{e} .
$$

Definition 2.2 Two MAPS with parameter matrices $\left(\boldsymbol{D}_{0}, \boldsymbol{D}_{1}\right)$ and $\left(S_{0}, S_{1}\right)$ are similar if there exist similarity transformations $S_{0}=$ $\boldsymbol{P} D_{0} \boldsymbol{P}^{-1}$ and $\boldsymbol{S}_{1}=\boldsymbol{P} D_{1} \boldsymbol{P}^{-1}$, for the same matrix $\boldsymbol{P}$, where $\boldsymbol{P} \vec{e}=\vec{e}$.

LEMMA 2.3 Two similar MAPs have stochastically equivalent interval stationary processes.

Proof Consider two similar MAPs $\left(D_{0}, D_{1}\right)$ and $\left(S_{0}, S_{1}\right)$ with the similarity transformation matrix $\boldsymbol{P}$. The invariant probability vector for the time stationary process $\vec{\pi}_{s}$ is given by $\vec{\pi}_{D} \boldsymbol{P}^{-1}$, and that for the interval stationary version is given by $\vec{\phi}_{S}=\vec{\phi}_{D} \boldsymbol{P}^{-1}$. Using the similarity transformations in Definition 2.2 , the requirements for SE are readily seen to be fulfilled.

\section{EXAMPLES}

We now give some examples of stochastically equivalent point processes derived from similar two-state MAPs. In the examples we 
draw upon the results derived in the Appendix concerning the valid region for similarity transformations of a given two-state MAP.

Example 3.1 The stochastical equivalence of the $H_{2}$ renewal process and the IPP is well known. In an MAP context we can express this by the similarity of the MAP representations of the $\mathrm{H}_{2}$ renewal process:

$$
\boldsymbol{D}_{0}=\left[\begin{array}{cc}
-\mu_{1} & 0 \\
0 & -\mu_{2}
\end{array}\right], \quad \boldsymbol{D}_{1}=\left[\begin{array}{cc}
\mu_{1} p & \mu_{1}(1-p) \\
\mu_{2} p & \mu_{2}(1-p)
\end{array}\right]
$$

and the IPP,

$$
\begin{aligned}
& \boldsymbol{S}_{0}=\left[\begin{array}{cc}
-\frac{\left(p \mu_{1}^{2}+(1-p) \mu_{2}^{2}\right)}{\mu_{1} p+\mu_{2}(1-p)} & \frac{p(1-p)\left(\mu_{1}-\mu_{2}\right)^{2}}{\mu_{1} p+\mu_{2}(1-p)} \\
\frac{\mu_{1} \mu_{2}}{\mu_{1} p+\mu_{2}(1-p)} & -\frac{\mu_{1} \mu_{2}}{\mu_{1} p+\mu_{2}(1-p)}
\end{array}\right], \\
& \boldsymbol{S}_{1}=\left[\begin{array}{cc}
\mu_{1} p+\mu_{2}(1-p) & 0 \\
0 & 0
\end{array}\right],
\end{aligned}
$$

which are related through $\boldsymbol{S}_{i}=\boldsymbol{P} \boldsymbol{D}_{i} \boldsymbol{P}^{-1}, i=0,1$ with

$$
\begin{aligned}
\boldsymbol{P} & =\left[\begin{array}{cc}
p & 1-p \\
\frac{-\mu_{2}}{\mu_{1}-\mu_{2}} & \frac{\mu_{1}}{\mu_{1}-\mu_{2}}
\end{array}\right], \\
\boldsymbol{P}^{-1} & =\left[\begin{array}{cc}
\frac{\mu_{1}}{\mu_{1} p+\mu_{2}(1-p)} & \frac{\left(\mu_{2}-\mu_{1}\right)(1-p)}{\mu_{1} p+\mu_{2}(1-p)} \\
\frac{\mu_{2}}{\mu_{1} p+\mu_{2}(1-p)} & \frac{-\left(\mu_{2}-\mu_{1}\right) p}{\mu_{1} p+\mu_{2}(1-p)}
\end{array}\right] .
\end{aligned}
$$

Another similarity transformation transforms the $\mathrm{H}_{2}$ renewal process into a renewal process of a mixture of Generalized Erlang (GE)distributions. The MAP representing the GE renewal process

$$
\boldsymbol{T}_{0}=\left[\begin{array}{cc}
-\mu_{1} & \left(\mu_{1}-\mu_{2}\right)(1-p) \\
0 & -\mu_{2}
\end{array}\right], \quad \boldsymbol{T}_{1}=\left[\begin{array}{cc}
\mu_{1} p+\mu_{2}(1-p) & 0 \\
\mu_{2} & 0
\end{array}\right],
$$


is related to $\left(D_{0}, D_{1}\right)$ through $T_{i}=Q D_{i} Q^{-1}, i=0,1$ with

$$
\boldsymbol{Q}=\left[\begin{array}{cc}
p & 1-p \\
0 & 1
\end{array}\right], \quad \boldsymbol{Q}^{-1}=\left[\begin{array}{cc}
\frac{1}{p} & \frac{-(1-p)}{p} \\
0 & 1
\end{array}\right]
$$

Example 3.2 In [3] it was shown that for every two-state MMPP (or SPP) point process there exists a stochastically equivalent Markov Switched Poisson Process (MSPP) point process. The MSPP can be parameterized as follows:

$$
\boldsymbol{D}_{0}=\left[\begin{array}{cc}
-\mu_{1} & 0 \\
0 & -\mu_{2}
\end{array}\right], \quad \boldsymbol{D}_{1}=\left[\begin{array}{ll}
\mu_{1} p_{1} & \mu_{1}\left(1-p_{1}\right) \\
\mu_{2} p_{2} & \mu_{2}\left(1-p_{2}\right)
\end{array}\right] .
$$

Also here SE can be expressed as a similarity transformation. Let the SPP be parameterized by

$$
S_{0}=\left[\begin{array}{cc}
-r_{1}-\lambda_{1} & r_{1} \\
r_{2} & -r_{2}-\lambda_{2}
\end{array}\right], \quad S_{1}=\left[\begin{array}{cc}
\lambda_{1} & 0 \\
0 & \lambda_{2}
\end{array}\right],
$$

where

$$
\begin{gathered}
\lambda_{1}=\frac{p_{1} \mu_{1}+\left(1-p_{2}\right) \mu_{2}+\sqrt{\left(p_{1} \mu_{1}-\left(1-p_{2}\right) \mu_{2}\right)^{2}+4 \mu_{1} \mu_{2}\left(1-p_{1}\right) p_{2}}}{2}, \\
\lambda_{2}=\frac{p_{1} \mu_{1}+\left(1-p_{2}\right) \mu_{2}-\sqrt{\left(p_{1} \mu_{1}-\left(1-p_{2}\right) \mu_{2}\right)^{2}+4 \mu_{1} \mu_{2}\left(1-p_{1}\right) p_{2}}}{2}, \\
r_{1}=\frac{\left(\left(1-p_{1}\right) \mu_{1}+p_{2} \mu_{2}\right) \lambda_{1}-\left(\left(1-p_{1}\right)+p_{2}\right) \mu_{1} \mu_{2}}{\lambda_{1}-\lambda_{2}}, \\
r_{2}=\frac{\left(\left(1-p_{1}\right)+p_{2}\right) \mu_{1} \mu_{2}-\left(\left(1-p_{1}\right) \mu_{1}+p_{2} \mu_{2}\right) \lambda_{2}}{\lambda_{1}-\lambda_{2}} .
\end{gathered}
$$

Then $S_{i}=Q D_{i} Q^{-1}, i=0,1$ with

$$
\boldsymbol{Q}=\left[\begin{array}{ll}
q_{1} & 1-q_{1} \\
q_{2} & 1-q_{2}
\end{array}\right], \quad \boldsymbol{Q}^{-1}=\frac{1}{q_{1}-q_{2}}\left[\begin{array}{cc}
1-q_{2} & -\left(1-q_{1}\right) \\
-q_{2} & q_{1}
\end{array}\right],
$$

where

$$
\begin{aligned}
& q_{1}=\frac{\left(1+p_{2}\right) \mu_{2}-p_{1} \mu_{1}+\sqrt{\left(p_{1} \mu_{1}-\left(1-p_{2}\right) \mu_{2}\right)^{2}+4 \mu_{1} \mu_{2}\left(1-p_{1}\right) p_{2}}}{2\left(\mu_{2}-\mu_{1}\right)}, \\
& q_{2}=\frac{\left(1+p_{2}\right) \mu_{2}-p_{1} \mu_{1}-\sqrt{\left(p_{1} \mu_{1}-\left(1-p_{2}\right) \mu_{2}\right)^{2}+4 \mu_{1} \mu_{2}\left(1-p_{1}\right) p_{2}}}{2\left(\mu_{2}-\mu_{1}\right)} .
\end{aligned}
$$




\section{Remarks}

- As noted in [3], a stochastically equivalent MSPP point process for a given SPP point process can always be found, while the converse is true only when the MSPP satisfies the condition $p_{1}\left(1-p_{2}\right) \geq$ $\left(1-p_{1}\right) p_{2}$.

- With the parameterization used in this example, the stochastical equivalence of the MSPP and SPP point processes can be seen as a generalization of the SE of the IPP and $H_{2}$ renewal process in the previous example. This is seen by setting $p_{1}=p_{2}=p$.

\section{FINAL REMARKS}

Similarity is an attractive property since it is relatively easy to check whether two matrices are similar by solving the Sylvester equation ([2], p. 310). However, it is clear that similarity is not a necessary condition for the SE of the interval stationary point processes induced by MAPs. Even for non-trivial two-state MAPs, similarity is not a necessary condition for SE, as the next example demonstrates. The similarity of the $\mathrm{H}_{2}$ renewal process and the IPP and that of the MSPP and the SPP are thus by no means automatic.

Example 4.1 We consider the following two MAPs with stochastically equivalent stationary point processes (the SE can readily be verified using results from [1]):

$$
\begin{gathered}
\boldsymbol{D}_{0}=\left[\begin{array}{cc}
-26.001600 & 2.159218 \\
0.919591 & -16.998400
\end{array}\right], D_{1}=\left[\begin{array}{cc}
16.001600 & 7.840782 \\
4.080409 & 11.998400
\end{array}\right], \\
D=\left[\begin{array}{cc}
-10.000000 & 10.000000 \\
5.000000 & -5.000000
\end{array}\right], \\
S_{0}=\left[\begin{array}{cc}
-26.000000 & 2.000000 \\
1.000000 & -17.000000
\end{array}\right], S_{1}=\left[\begin{array}{ll}
16.000000 & 8.000000 \\
4.000000 & 12.000000
\end{array}\right], \\
S=\left[\begin{array}{cc}
-10.000000 & 10.000000 \\
5.000000 & -5.000000
\end{array}\right] .
\end{gathered}
$$


The two MAPs are not similar since

$$
S=Q D Q^{-1}, \quad S_{0}=Q_{0} D_{0} Q_{0}^{-1}, \quad S_{1}=Q_{1} D_{1} Q_{1}^{-1}
$$

with

$$
\begin{gathered}
Q=\left[\begin{array}{ll}
1.000000 & 0.000000 \\
0.000000 & 1.000000
\end{array}\right], \quad Q_{0}=\left[\begin{array}{ll}
0.000000 & 0.222183 \\
0.102158 & 1.000000
\end{array}\right], \\
Q_{1}=\left[\begin{array}{cc}
0.000000 & -1.999200 \\
-1.019694 & 1.000000
\end{array}\right] .
\end{gathered}
$$

\section{Acknowledgments}

The first author would like to acknowledge the financial support of the Technical Research Council of Denmark under grant no. 9400273.

\section{References}

[1] A.T. Andersen and B.F. Nielsen. On the use of second order descriptors to characterize MAPs. In COST 257 TD(97)050, pp. 1-24. COST, September 1997.

[2] N.J. Higham. Accuracy and Stability of Numerical Algorithms. SIAM, Philadelphia, 1996.

[3] D. Liu and M.F. Neuts. Counter-examples involving Markovian arrival processes. Commun. Statist. -Stochastic Models, 7(3): 499-509, 1991.

[4] D.M. Lucantoni. The BMAP/G/1 queue: A tutorial. In L. Donatiello and R. Nelson (Eds.), Models and Techniques for Performance Evaluation of Computer and Communication Systems, Springer Verlag, Berlin, 1993.

[5] M.F. Neuts. Structured Stochastic Matrices of M/G/1 Type and Their Applications, Probability: Pure and Applied, In Vol. 5, Marcel Dekker, Inc., 1989.

\section{APPENDIX}

\section{Similarity Transformations of Two-State MAPs}

In this appendix we identify classes of two-state MAPs which arise by similarity transformations. We are only interested in MAPs where the two-state description is minimal (i.e., MAPs that cannot be reduced to a Poisson process). As shown in Section 2, similar MAPs are stochastically equivalent.

Given an MAP minimally represented by $D_{0}$ and $D_{1}$, where $D_{0}$ is a sub-generator and $D=\left(D_{0}+D_{1}\right)$ is a generator, the goal is to 
find all $S_{0}=P D_{0} P^{-1}$ and $S_{1}=P D_{1} P^{-1}$ such that $S_{0}$ and $S_{1}$ represent a MAP.

Let $\vec{\pi}$ be the time stationary probability vector for $\boldsymbol{D}$. Then it is easy to see that the counting processes of the MAPs given by $\boldsymbol{D}_{0}, \boldsymbol{D}_{1}$ and $S_{0}, S_{1}$ are identical:

$$
\vec{\pi} \exp \left(\left(\boldsymbol{D}_{0}+z \boldsymbol{D}_{1}\right) t\right) \vec{e}=\vec{\pi} \boldsymbol{P}^{-1} \exp \left(\left(\boldsymbol{S}_{0}+z \boldsymbol{S}_{1}\right) t\right) \boldsymbol{P} \vec{e},
$$

where $\boldsymbol{P} \vec{e}=\vec{e}$.

For the two-state MAP, the fact that $S=S_{0}+S_{1}$ is a generator can readily be seen to limit $\boldsymbol{P}$ to the structure below when $p_{2} \neq p_{1}$ :

$$
\boldsymbol{P}=\left[\begin{array}{ll}
p_{1} & 1-p_{1} \\
p_{2} & 1-p_{2}
\end{array}\right], \quad \boldsymbol{P}^{-1}=\frac{1}{p_{1}-p_{2}}\left[\begin{array}{cc}
1-p_{2} & -\left(1-p_{1}\right) \\
-p_{2} & p_{1}
\end{array}\right] .
$$

Without loss of generality we restrict ourselves to the case $p_{1}>p_{2}$ since the case $p_{2}>p_{1}$ corresponds only to a relabeling of the two states in $S_{0}$ and $S_{1}$ with $p_{1}>p_{2}$.

In the following we parameterize $D_{0}$ and $D_{1}$ as

$$
\boldsymbol{D}_{0}=d\left[\begin{array}{cc}
-1 & q_{1} \\
c q_{2} & -c
\end{array}\right], \quad \boldsymbol{D}_{1}=d\left[\begin{array}{cc}
h_{1}\left(1-q_{1}\right) & \left(1-h_{1}\right)\left(1-q_{1}\right) \\
c h_{2}\left(1-q_{2}\right) & c\left(1-h_{2}\right)\left(1-q_{2}\right)
\end{array}\right],
$$

where $d>0,0<c \leq 1,0 \leq q_{1} \leq 1,0 \leq q_{2} \leq 1,0 \leq h_{1} \leq 1,0 \leq h_{2} \leq 1$, $q_{1}+\left(1-h_{1}\right)>0$ and $q_{2}+h_{2}>0$. Straightforward, but tedious, calculations lead to the expressions

$$
\boldsymbol{S}_{0}=\left[\begin{array}{ll}
S_{0_{11}} & S_{0_{12}} \\
S_{0_{21}} & S_{0_{22}}
\end{array}\right], \quad \boldsymbol{S}_{1}=\left[\begin{array}{ll}
S_{1_{11}} & S_{1_{12}} \\
S_{1_{21}} & S_{1_{22}}
\end{array}\right]
$$

where

$$
\begin{aligned}
S_{0_{11}}= & \frac{d}{p_{1}-p_{2}}\left(-p_{2} p_{1}\left(c\left(1-q_{2}\right)-\left(1-q_{1}\right)\right)\right. \\
& \left.+c\left(1-q_{2}\right) p_{2}-\left(1+q_{2} c\right) p_{1}+q_{2} c\right), \\
S_{0_{12}}= & \frac{d}{p_{1}-p_{2}}\left(p_{1}^{2}\left(c\left(1-q_{2}\right)-\left(1-q_{1}\right)\right)+\left(1-c+2 q_{2} c\right) p_{1}-q_{2} c\right), \\
S_{0_{21}}= & \frac{d}{p_{1}-p_{2}}\left(-p_{2}^{2}\left(c\left(1-q_{2}\right)-\left(1-q_{1}\right)\right)-\left(1-c+2 q_{2} c\right) p_{2}+q_{2} c\right), \\
S_{0_{22}}= & \frac{d}{p_{1}-p_{2}}\left(p_{2} p_{1}\left(c\left(1-q_{2}\right)-\left(1-q_{1}\right)\right)-c\left(1-q_{2}\right) p_{1}\right. \\
& \left.+\left(1+q_{2} c\right) p_{2}-q_{2} c\right)
\end{aligned}
$$


and

$$
\begin{aligned}
S_{1_{11}}= & \frac{d}{p_{1}-p_{2}}\left(p_{2} p_{1}\left(c\left(1-q_{2}\right)-\left(1-q_{1}\right)\right)-c\left(1-q_{2}\right) p_{2}\right. \\
& \left.+\left(\left(1-q_{1}\right) h_{1}-c\left(1-q_{2}\right) h_{2}\right) p_{1}+c\left(1-q_{2}\right) h_{2}\right), \\
S_{1_{12}}= & \frac{d}{p_{1}-p_{2}}\left(-p_{1}^{2}\left(c\left(1-q_{2}\right)-\left(1-q_{1}\right)\right)+\left(c\left(1-q_{2}\right)\right.\right. \\
& \left.\left.+c\left(1-q_{2}\right) h_{2}-\left(1-q_{1}\right) h_{1}\right) p_{1}-c\left(1-q_{2}\right) h_{2}\right), \\
S_{1_{21}}= & \frac{d}{p_{1}-p_{2}}\left(p_{2}^{2}\left(c\left(1-q_{2}\right)-\left(1-q_{1}\right)\right)-\left(c\left(1-q_{2}\right)\right.\right. \\
& \left.\left.+c\left(1-q_{2}\right) h_{2}-\left(1-q_{1}\right) h_{1}\right) p_{2}+c\left(1-q_{2}\right) h_{2}\right), \\
S_{1_{22}}= & \frac{d}{p_{1}-p_{2}}\left(-p_{2} p_{1}\left(c\left(1-q_{2}\right)-\left(1-q_{1}\right)\right)+c\left(1-q_{2}\right) p_{1}\right. \\
& \left.-\left(\left(1-q_{1}\right) h_{1}-c\left(1-q_{2}\right) h_{2}\right) p_{2}-c\left(1-q_{2}\right) h_{2}\right) .
\end{aligned}
$$

The valid similarity transformations are those that make $S_{0_{11}}<0$, $S_{0_{22}}<0, S_{0_{12}} \geq 0, S_{0_{21}} \geq 0$ and all elements in $S_{1}$ non-negative with at least one non-zero element. Recall that $S=S_{0}+S_{1}$ and $S \vec{e}=\overrightarrow{0}$.

The following quadratic expressions are important for sign behavior:

$$
\begin{aligned}
f(x)_{S_{0}}= & x^{2}\left(c\left(1-q_{2}\right)-\left(1-q_{1}\right)\right)+\left(1-c+2 q_{2} c\right) x-q_{2} c, \\
f(x)_{S_{1}}= & -x^{2}\left(c\left(1-q_{2}\right)-\left(1-q_{1}\right)\right)+\left(c\left(1-q_{2}\right)\right. \\
& \left.\left.+c\left(1-q_{2}\right) h_{2}-\left(1-q_{1}\right) h_{1}\right) x-c\left(1-q_{2}\right) h_{2}\right) .
\end{aligned}
$$

The roots of $f(x)_{S_{0}}$ are found to be

$$
\begin{aligned}
& \rho_{1}=\frac{1}{2\left(c\left(1-q_{2}\right)-\left(1-q_{1}\right)\right)}\left(c-1-2 q_{2} c+\sqrt{(1-c)^{2}+4 q_{1} q_{2} c}\right), \\
& \rho_{2}=\frac{1}{2\left(c\left(1-q_{2}\right)-\left(1-q_{1}\right)\right)}\left(c-1-2 q_{2} c-\sqrt{(1-c)^{2}+4 q_{1} q_{2} c}\right) .
\end{aligned}
$$

The roots of $f(x)_{S_{1}}$ are

$$
\begin{aligned}
\sigma_{1} & =\frac{c\left(1-q_{2}\right)\left(1+h_{2}\right)-\left(1-q_{1}\right) h_{1}}{2\left(c\left(1-q_{2}\right)-\left(1-q_{1}\right)\right)} \\
+ & \frac{\sqrt{\left(c\left(1-q_{2}\right)\left(1-h_{2}\right)-\left(1-q_{1}\right) h_{1}\right)^{2}+4 c\left(1-q_{1}\right)\left(1-q_{2}\right) h_{2}\left(1-h_{1}\right)}}{2\left(c\left(1-q_{2}\right)-\left(1-q_{1}\right)\right)}
\end{aligned}
$$




$$
\begin{aligned}
\sigma_{2} & =\frac{c\left(1-q_{2}\right)\left(1+h_{2}\right)-\left(1-q_{1}\right) h_{1}}{2\left(c\left(1-q_{2}\right)-\left(1-q_{1}\right)\right)} \\
- & \frac{\sqrt{\left(c\left(1-q_{2}\right)\left(1-h_{2}\right)-\left(1-q_{1}\right) h_{1}\right)^{2}+4 c\left(1-q_{1}\right)\left(1-q_{2}\right) h_{2}\left(1-h_{1}\right)}}{2\left(c\left(1-q_{2}\right)-\left(1-q_{1}\right)\right)} .
\end{aligned}
$$

From the roots and signs of $f(x)_{S_{0}}$ and $f(x)_{S_{1}}$ one can find the parameter values $\left(p_{1}, p_{2}\right)$ that make the off-diagonal elements of $\boldsymbol{S}_{0}$ and $S_{1}$ non-negative.

The restrictions on $\left(p_{1}, p_{2}\right)$ imposed by the requirements on the diagonal elements of $S_{0}$ can be found from the elementwise inequality $S_{0} \vec{e} \leq \overrightarrow{0}$ which implies

$$
\frac{p_{i}}{k_{d}} \leq 1 \quad(i=1,2) \quad \text { where } k_{d}=\frac{c\left(1-q_{2}\right)}{c\left(1-q_{2}\right)-\left(1-q_{1}\right)} .
$$

These restrictions are imposed by the non-negativity of the diagonal elements of $\boldsymbol{S}_{1}$. Unlike the restrictions found above, valid values of $p_{1}$ and $p_{2}$ in the present case are not mutually independent. From the above it can be seen that $S_{1_{11}} \geq 0$ implies

$$
\begin{aligned}
& p_{2}\left(p_{1}\left(c\left(1-q_{2}\right)-\left(1-q_{1}\right)\right)-c\left(1-q_{2}\right)\right) \\
& \quad \geq\left(c\left(1-q_{2}\right) h_{2}-\left(1-q_{1}\right) h_{1}\right) p_{1}-c\left(1-q_{2}\right) h_{2} .
\end{aligned}
$$

This inequality has the form $p_{2} g_{1}\left(p_{1}\right) \geq g_{2}\left(p_{1}\right)$. Let

$$
\begin{aligned}
b_{1}\left(p_{1}\right) & =\frac{g_{2}\left(p_{1}\right)}{g_{1}\left(p_{1}\right)} \\
& =\frac{\left(c\left(1-q_{2}\right) h_{2}-\left(1-q_{1}\right) h_{1}\right) p_{1}-c\left(1-q_{2}\right) h_{2}}{p_{1}\left(c\left(1-q_{2}\right)-\left(1-q_{1}\right)\right)-c\left(1-q_{2}\right)} .
\end{aligned}
$$

It can be seen that $S_{1_{22}} \geq 0$ implies

$$
\begin{aligned}
& -p_{2}\left(p_{1}\left(c\left(1-q_{2}\right)-\left(1-q_{1}\right)\right)+\left(1-q_{1}\right) h_{1}-c\left(1-q_{2}\right) h_{2}\right) \\
& \quad \geq c\left(1-q_{2}\right) h_{2}-c\left(1-q_{2}\right) p_{1} .
\end{aligned}
$$

This inequality has the form $p_{2} e_{1}\left(p_{1}\right) \leq e_{2}\left(p_{1}\right)$. Let

$$
\begin{aligned}
b_{2}\left(p_{1}\right) & =\frac{e_{2}\left(p_{1}\right)}{e_{1}\left(p_{1}\right)} \\
& =\frac{c\left(1-q_{2}\right) p_{1}-c\left(1-q_{2}\right) h_{2}}{p_{1}\left(c\left(1-q_{2}\right)-\left(1-q_{1}\right)\right)+\left(1-q_{1}\right) h_{1}-c\left(1-q_{2}\right) h_{2}} .
\end{aligned}
$$


Clearly the similarity transformations we are looking for are those with parameter values $\left(p_{1}, p_{2}\right)$ that obey all the restrictions listed above.

To summarize, we have the following bounds on $p_{1}$ and $p_{2}$. If for any of the intervals below $a>b$, then $[a, b] \in \emptyset$.

The case $c\left(1-q_{2}\right)-\left(1-q_{1}\right)>0$ :

$$
\begin{aligned}
& p_{1} \in\left[\max \left(\rho_{1}, \sigma_{2}\right), \min \left(\sigma_{1}, k_{d}\right)\right] \\
& \text { if } p_{1} \leq \frac{c\left(1-q_{2}\right) h_{2}-\left(1-q_{1}\right) h_{1}}{c\left(1-q_{2}\right)-\left(1-q_{1}\right)}, \\
& \quad p_{2} \in\left[\max \left(\rho_{2}, b_{2}\left(p_{1}\right)\right), \min \left(\rho_{1}, \sigma_{2}, b_{1}\left(p_{1}\right)\right)\right] \\
& \text { else } \\
& \qquad p_{2} \in\left[\rho_{2}, \min \left(\rho_{1}, \sigma_{2}, b_{1}\left(p_{1}\right), b_{2}\left(p_{1}\right)\right)\right] .
\end{aligned}
$$

The case $c\left(1-q_{2}\right)-\left(1-q_{1}\right)<0$ :

$$
\begin{aligned}
& p_{1} \in\left[\max \left(\rho_{1}, \sigma_{2}\right), \rho_{2}\right] \\
& \text { if } p_{1} \leq \frac{c\left(1-q_{2}\right) h_{2}-\left(1-q_{1}\right) h_{1}}{c\left(1-q_{2}\right)-\left(1-q_{1}\right)}, \\
& \qquad p_{2} \in\left[\max \left(k_{d}, \sigma_{1}\right), \min \left(\rho_{1}, \sigma_{2}, b_{1}\left(p_{1}\right), b_{2}\left(p_{1}\right)\right)\right] \\
& \text { else } \\
& \qquad p_{2} \in\left[\max \left(k_{d}, \sigma_{1}, b_{2}\left(p_{1}\right)\right), \min \left(\rho_{1}, \sigma_{2}, b_{1}\left(p_{1}\right)\right)\right] .
\end{aligned}
$$

Example A.1 In the case $h_{1}=h_{2}$ the MAP is readily identified as a two-state $\mathrm{PH}$ - renewal process. Letting $h=h_{1}=h_{2}$, we find that

$$
\begin{aligned}
\sigma_{1} & =k_{d}=\frac{c\left(1-q_{2}\right)}{c\left(1-q_{2}\right)-\left(1-q_{1}\right)}, \\
\sigma_{2} & =h, \quad b_{1}\left(p_{1}\right)=h, \\
b_{2}\left(p_{1}\right) & =k_{d}=\frac{c\left(1-q_{2}\right)}{c\left(1-q_{2}\right)-\left(1-q_{1}\right)} .
\end{aligned}
$$

This yields substantial simplifications of the valid region for $p_{1}$ and $p_{2}$.

The case $c\left(1-q_{2}\right)-\left(1-q_{1}\right)>0$ :

$$
p_{1} \in\left[\max \left(\rho_{1}, h\right), k_{d}\right] \quad \text { and } \quad p_{2} \in\left[\rho_{2}, \min \left(\rho_{1}, h\right)\right] .
$$


The case $c\left(1-q_{2}\right)-\left(1-q_{1}\right)<0$ :

$$
p_{1} \in\left[\max \left(\rho_{1}, h\right), \rho_{2}\right] \quad \text { and } \quad p_{2} \in\left[k_{d}, \min \left(\rho_{1}, h\right)\right] .
$$

Example A.2 In the case $h_{1}=1$ and $h_{2}=0$ the MAP $\left(\boldsymbol{D}_{0}, \boldsymbol{D}_{1}\right)$ is readily identified as the two-state MMPP (or SPP). In this case we have:

the case $c\left(1-q_{2}\right)-\left(1-q_{1}\right)>0: \sigma_{1}=1, \sigma_{2}=0$, the case $c\left(1-q_{2}\right)-\left(1-q_{1}\right)<0: \sigma_{1}=0, \sigma_{2}=1$,

$$
\begin{aligned}
& b_{1}\left(p_{1}\right)=\frac{-\left(1-q_{1}\right) p_{1}}{p_{1}\left(c\left(1-q_{2}\right)-\left(1-q_{1}\right)\right)-c\left(1-q_{2}\right)}, \\
& b_{2}\left(p_{1}\right)=\frac{c\left(1-q_{2}\right) p_{1}}{p_{1}\left(c\left(1-q_{2}\right)-\left(1-q_{1}\right)\right)+\left(1-q_{1}\right)} .
\end{aligned}
$$




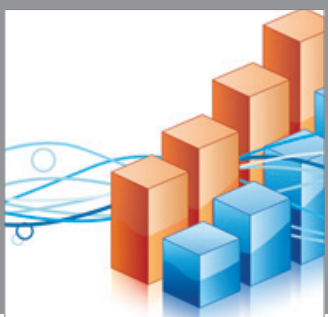

Advances in

Operations Research

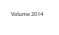

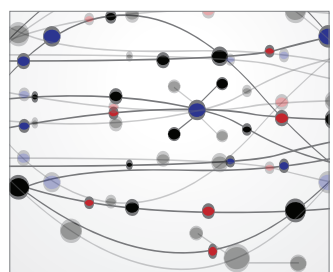

\section{The Scientific} World Journal
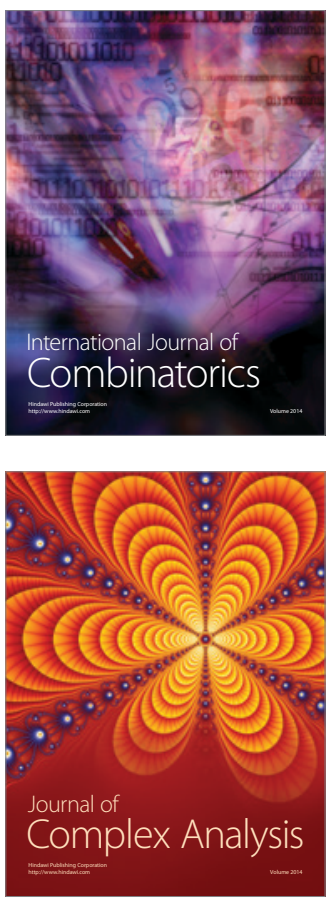

International Journal of

Mathematics and

Mathematical

Sciences
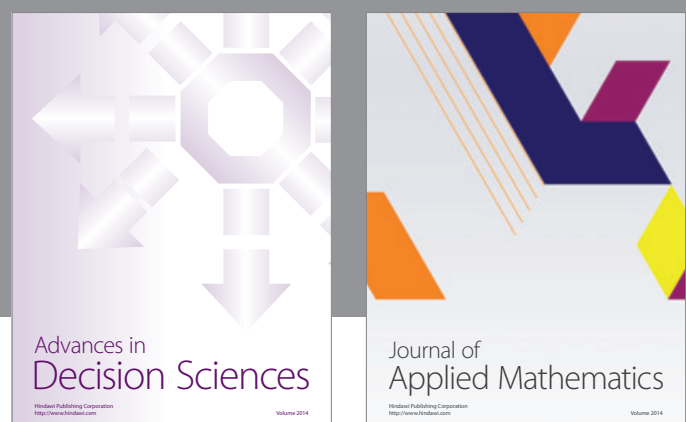

Journal of

Applied Mathematics
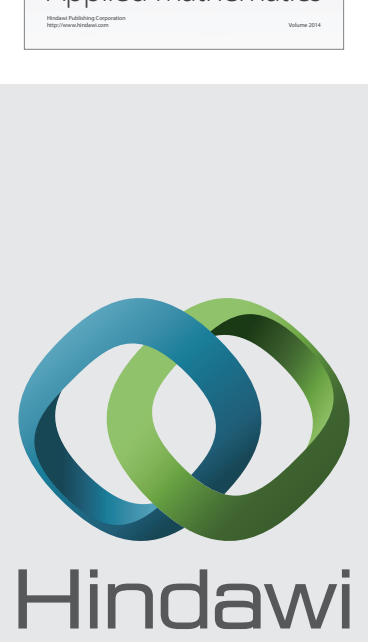

Submit your manuscripts at http://www.hindawi.com
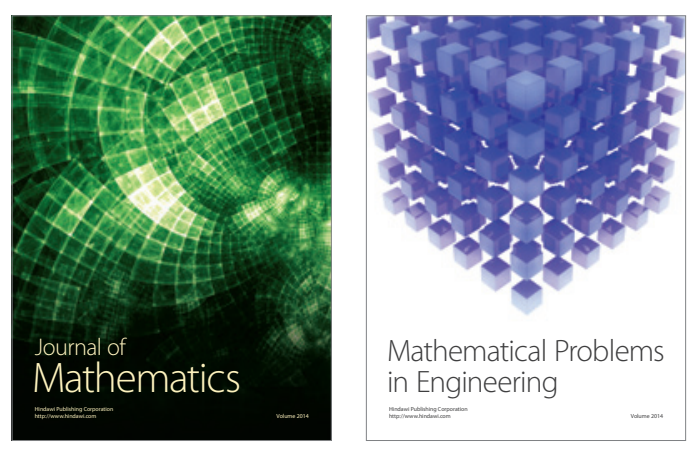

Mathematical Problems in Engineering
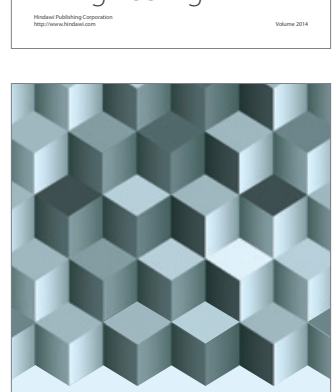

Journal of

Function Spaces
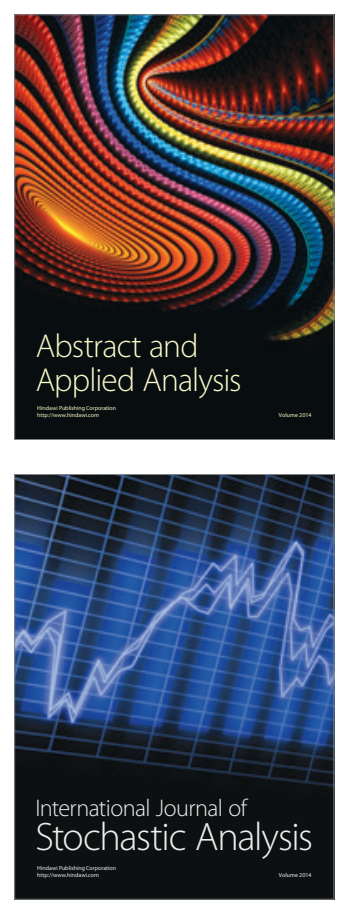

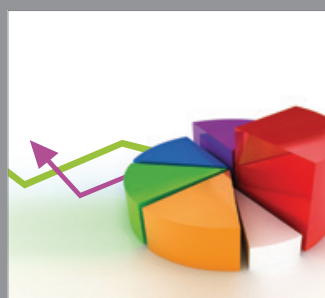

ournal of

Probability and Statistics

Promensencen
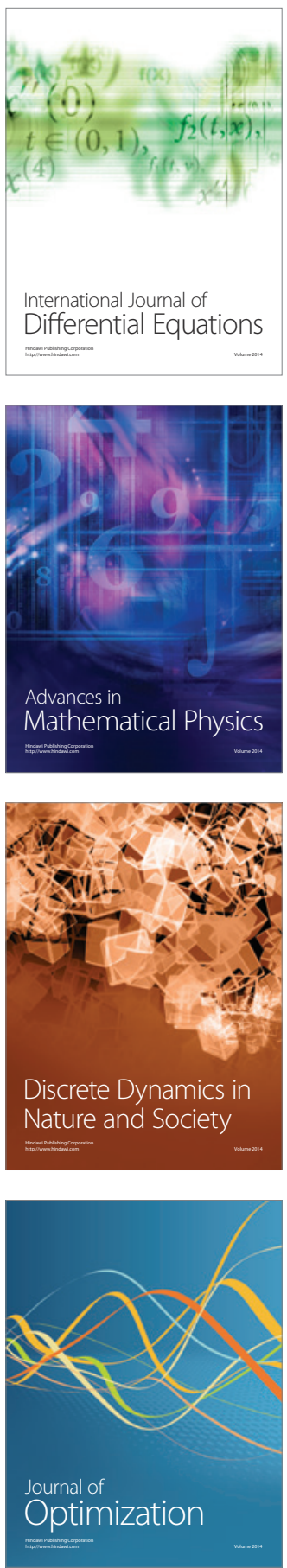